\title{
Alinhamento postural e qualidade vocal em cantores
}

\author{
Singers" postural alignment and vocal quality
}

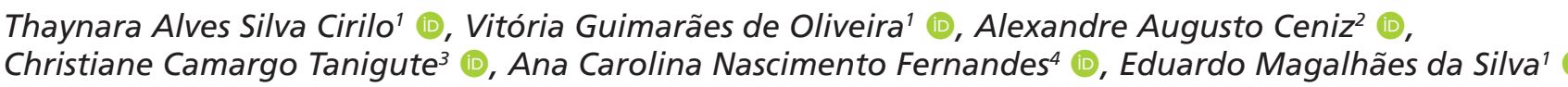

\section{RESUMO}

Objetivo: Identificar e descrever as alterações de postura e de qualidade de voz em um grupo de cantores populares. Métodos: Série de casos com nove voluntários (seis mulheres e três homens) com idade média de $28,3 \pm 6,4$ anos. A avaliação postural foi realizada pelo software SAPO. A avaliação perceptivoauditiva da voz foi realizada por juízes, utilizando o VPAS-PB, e a análise acústica com o software PRAAT. Resultados: Os tempos máximos fonatórios variaram entre 7,1 e 22,0 s. A frequência fundamental entre 104,7 e $247,7 \mathrm{~Hz}$, mostrando-se adequada ao sexo e à idade dos participantes. A intensidade, o jitter e o shimmer variaram dentro dos valores de referência, respectivamente, 59,8 e $68,6 \mathrm{~dB}, 0,110$ e $0,306 \%$ e 0,903 a 2,673 dB. Enquanto a proporção harmônico-ruído ficou acima dos valores referência para ambos os sexos, entre 18,1 e 31,5 dB. A avaliação perceptivoauditiva da voz definiu ajustes não neutros à fonação, com nove participantes apresentando soprosidade; cinco, aspereza, e dois, crepitação, com presença de suporte respiratório inadequado. Todos os participantes apresentaram alterações e/ou adaptações posturais. Conclusão: Foram identificados e descritos ajustes não neutros à fonação, definindo alterações de qualidade vocal em um grupo de cantores, no qual também se observou que os participantes apresentaram adaptações e/ou alterações posturais, o que pode sugerir que estes ajustes coocorrem no grupo avaliado.

Palavras-chave: Qualidade da voz; Canto; Voz; Postura; Equilíbrio postural

\begin{abstract}
Purpose: To identify and describe changes in posture and voice quality in a group of popular singers. Methods: Series of cases with nine volunteers (six women and three men) with a mean age of $28.3 \pm 6.4$ years. Postural assessment was performed using the SAPO software. The auditory-perceptual assessment of the voice was performed by judges using the VPAS-PB, and the acoustic analysis was carried out with the PRAAT software. Results: The maximum phonation times varied between 7.1 and $22.0 \mathrm{sec}$. The fundamental frequency between 104.7 and $247.7 \mathrm{~Hz}$, adequate to the gender and age of the participants. The intensity, jitter and shimmer varied within the reference values, respectively, 59.8 and $68.6 \mathrm{~dB}, 0.110$ and $0.306 \%$ and 0.903 to $2.673 \mathrm{~dB}$. While the harmonic-noise ratio was above the reference values for both sexes, between 18.1 and $31.5 \mathrm{~dB}$. The auditory-perceptual assessment of the voice defined non-neutral phonation adjustments, with nine participants presenting breathy; five, harsh; and two, creaky voices, with the presence of inadequate respiratory support. All participants showed postural changes and / or adaptations. Conclusion: Non-neutral adjustments to phonation were identified and described, defining alterations in vocal quality in a group of singers, in which participants also presented adaptations and / or postural alterations, which may suggest that these adjustments cooccur in the group evaluated.
\end{abstract}

Keywords: Voice quality; Singing; Voice; Posture; Posture balance

\footnotetext{
Trabalho realizado no Laboratório de Ensino e Pesquisa em Otorrinolaringologia, Universidade de Brasília - UnB - Brasília (DF), Brasil.

${ }^{1}$ Coordenação de Fonoaudiologia, Faculdade de Ceilândia, Universidade de Brasília - UnB - Brasília (DF), Brasil.

${ }^{2}$ Academia BodyTech Lago Sul, Brasília (DF), Brasil.

${ }^{3}$ Coordenação de Fonoaudiologia, Pontifícia Universidade Católica de Goiás - PUC/GO - Goiânia (GO), Brasil.

${ }^{4}$ Programa de Pós-Graduação em Ciências Médicas, Universidade de Brasília - UnB - Brasília (DF), Brasil.

Conflito de interesses: Não.

Contribuição dos autores: TAS participou na coleta, interpretação, análise de dados e preparação do manuscrito; VGO participou na coleta, interpretação, análise de dados e preparação do manuscrito; AAC participou como coorientador do estudo na coleta e análise de dados e preparação do manuscrito; CCT participou na revisão crítica do manuscrito; ACNF participou como orientadora do estudo, interpretação, análise de dados e revisão crítica do manuscrito; EMS participou como orientador do estudo, interpretação, análise de dados, escrita e revisão crítica do manuscrito.
}

Financiamento: Nada a declarar.

Autor correspondente: Eduardo Magalhães da Silva. E-mail: edumagalhaes@unb.br

Recebido: Abril 03, 2021; Aceito: Setembro 16, 2021 


\section{INTRODUÇÃO}

O canto é uma atividade que requer treinamento, uso e adaptação do mecanismo da voz e um alinhamento postural apropriado. O controle da respiração é maior quando se assume uma postura adequada, que, além de permitir um melhor aproveitamento das cavidades de ressonância, inibe o aumento da tensão dos músculos do pescoço e favorece, de forma indireta, a produção de uma boa voz ${ }^{(1)}$.

O desequilíbrio postural pode ocorrer tanto devido à tensão exagerada, quanto à hipotonia e enfraquecimento muscular dos músculos responsáveis pelo alinhamento corporal. Quando a postura-padrão não atinge um estado de equilíbrio no espaço, pode provocar lesões ou desajustes às estruturas relacionadas a estes músculos, pois não terão sustentação para o desempenho de sua atividade, o que pode causar dor e desconforto ${ }^{(2)}$. Assim, o desalinhamento ou desequilíbrio da coluna vertebral, principalmente da área cervical, influencia diretamente a posição do osso hioide e indiretamente a posição da laringe (3) e, ainda que a avaliação postural não faça parte da formação do fonoaudiólogo, o estudo da fisiologia muscular é importante para a compreensão e análise das complexas interações entre os músculos que afetam a manutenção de um bom alinhamento postural ${ }^{(4)}$.

A postura ereta com ombros relaxados e queixo levemente abaixado é a mais adequada para a produção vocal por proporcionar uma livre movimentação da laringe e uma projeção do som sem bloqueio no trato vocal. Uma voz tensa decorre de uma ação muscular intrínseca e/ou extrínseca excessiva e, em geral, está acompanhada de uma postura característica, com cabeça anteriorizada e tensão nos músculos da cintura cervicoescapular $^{(5)}$. Essa postura inadequada, que deixa "marcas" em cada indivíduo, pode causar disfunção no funcionamento das pregas vocais, interferindo na respiração e a relação contrária também é válida ${ }^{(6)}$.

Refletir acerca dos trilhos anatômicos, definidos por Myers, e o questionamento sobre a comparação das relações posturais e estruturais, pode resultar em estratégias de tratamento que possibilitem reverter padrões corporais de compensação, levanta o conceito de "tensegridade", proposto por Fuller em $1975^{(7)}$, que sugere que a tração e a compressão combinadas proporcionam estabilidade e resistência, assegurando a integridade global de um corpo, ou seja, cria um "neutro" resiliente e estável em torno do qual ocorre o movimento.

Desta forma, o indivíduo que desenvolve um trabalho vocal deve ser considerado na sua totalidade, para que realmente possa se beneficiar da aplicação das técnicas vocais e corporais como recursos facilitadores para a sua produção vocal ${ }^{(8)}$.

Músicos exteriorizam uma acentuada concentração em torno de um objeto que não pode mudar de forma e a tendência de que o corpo se molde em torno do instrumento sólido é muito forte em todos os estilos de música ${ }^{(7)}$. Ao se extrapolar esta relação para os cantores, pode-se sugerir que o microfone pode ser um suporte à posição corporal, que favorecerá mudanças de postura ao longo de sua apresentação. Vale ressaltar que a postura é dinâmica e uma vez que as partes do corpo sofrem adaptações frequentes em resposta aos estímulos que recebe no decorrer do tempo, o corpo reflete as experiências vividas a cada momento ${ }^{(7)}$.

Em cantores, a associação entre alterações da voz e da postura pode ser mais expressiva ou manifesta, haja vista a utilização do corpo durante suas apresentações e a requisição de "adaptações", que podem requerer ajustes vocais e laríngeos para $\mathrm{o}$ atendimento à demanda vocal. Acreditamos que este estudo possa direcionar a atenção do fonoaudiólogo ao alinhamento postural biomecânico na busca de uma melhora na qualidade vocal de seu cliente. Assim, este estudo assumiu que alterações ou adaptações posturais poderiam coocorrer com alterações vocais e teve como objetivo identificar e descrever alterações de postura e de qualidade de voz em um grupo de cantores populares.

\section{MÉTODOS}

Estudo tipo série de casos realizado com nove cantores profissionais voluntários, sendo três do gênero masculino (32,0 $\pm 5,3$ anos de idade e $8,3 \pm 4,7$ anos de canto profissional) e seis do gênero feminino $(26,5 \pm 6,5$ anos de idade e $11,3 \pm 10,6$ anos de canto profissional) com idade média de $28,3 \pm 6,4$ anos e tempo médio de canto profissional de 10,3 $\pm 8,8$ anos.

Inicialmente, o estudo contou com 15 sujeitos que procuraram o laboratório de pesquisa de forma voluntária. Contudo, foram excluídos os dados de seis indivíduos por não atenderem aos critérios de elegibilidade, a saber: (1) estar em acompanhamento fonoaudiológico para alterações de fala e/ou voz durante a participação no estudo; (2) apresentarem alterações vocais de grau extremo, detectadas na avaliação perceptivoauditiva e/ou acústica e/ou (3) por não terem preenchido de forma completa ou legível os dados demográficos solicitados.

Todos os participantes possuíam exames laringoscópicos realizados nos seis meses que antecederam à coleta dos dados, com laudo de normalidade, emitido por médico otorrinolaringologista.

Foram coletadas informações pessoais, profissionais e de desenvolvimento e uso da voz cantada. As vozes foram gravadas em cabine acústica, com microfone unidirecional com resposta linear, sem condensador (Audio 50, Plantronics Inc., Califórnia, EUA), com taxa de amostragem de $44.100 \mathrm{~Hz}$, obedecendo-se à sequência: vogal /a/ sustentada em tempo máximo de fonação (TMF) e da frase foneticamente balanceada utilizada no protocolo Vocal Profile Analysis Scheme for Brazilian Portuguese (VPAS$\mathrm{PB})$ : “O objeto de estudo da fonética é essa complexa variável e poderosa face sonora da linguagem: a fala",

A avaliação perceptivoauditiva (APA) da voz foi realizada por três juízes com ampla experiência em avaliação da voz nos contextos clínico e de pesquisa, após sessões de treinamento e calibração, com cerca de duas horas cada, para que os juízes se familiarizassem com o instrumento de avaliação e seu preenchimento e tivessem o mesmo entendimento sobre os parâmetros em avaliação.

AAPA foi realizada por meio do VPAS-PB, protocolo baseado na descrição fonética da qualidade da voz, em termos de ajustes (settings), que incluem as dimensões: fonação (modo de vibração das pregas vocais), trato vocal (supralaríngeo e articulatório) e tensão (laríngea e trato vocal). Todas as dimensões são avaliadas a partir de uma configuração neutra (referência), na qual a vibração das pregas vocais é equilibrada (forças de adução e tensão longitudinal), a voz é produzida sem sussurro audível ou outros ruídos, as cavidades do trato vocal supralaríngeo não apresentam quaisquer graus de constrição ou expansão; a distância total entre as cordas vocais e os lábios é mantida intermediária sem efeitos de encurtamento ou alongamento, e as tensões laríngea e supralaríngea são moderadas ${ }^{(9)}$. 
Os examinadores não receberam qualquer informação quanto à faixa etária, presença de queixas vocais, modificações ou distúrbios da voz dos sujeitos pesquisados e foram orientados a utilizar fones de ouvido para ouvir as vozes em volume confortável e fazer o registro dos parâmetros que considerassem neutros (sem desvios) naquelas vozes. Em seguida, ouvi-las tantas vezes quantas julgassem necessário para avaliação dos parâmetros não-neutros (com desvios), quantificando-os em graus moderado (escores 1, 2 ou 3 ) ou extremo (escores 4, 5 ou 6). Além de identificarem as ocorrências de curto prazo (quebras, instabilidades, diplofonia e tremor).

Para a análise acústica da voz foi utilizado o software PRAAT (versão 6.1.16, https://www.fon.hum.uva.nl/praat/), no qual foram selecionados trechos de 3 a 5 segundos da emissão sustentada da vogal /a/ e foram extraídos, no módulo voice report, os valores de frequência fundamental (F0), jitter, shimmer e proporção harmônico/ruído (PHR).

A tomada das fotografias seguiu as recomendações do software SAPO (http://pesquisa.ufabc.edu.br/bmclab/sapo/), com um fio de prumo preso ao teto, com duas bolas de isopor distanciadas um metro uma da outra, coladas sobre o fio para posterior calibração da imagem. O sujeito foi posicionado de tal modo que ele e o fio de prumo ficassem num mesmo plano perpendicular ao eixo da câmera fotográfica digital de 13.0 megapixels (Nikon, Coolpix L820), posicionada em tripé, nivelada a altura de $1,20 \mathrm{~m}$, paralela ao chão, situada a $2,35 \mathrm{~m}$ de distância do participante.

As tomadas fotográficas foram realizadas nas vistas anterior, posterior e lateral esquerda, com os participantes em traje de banho, com as referências ósseas, para os cálculos angulares, marcadas com bolas de isopor, atendendo ao protocolo SAPO, nos pontos anatômicos descritos na Figura 1. A marcação dos pontos anatômicos foi realizada por professor de Educação Física treinado, com ampla experiência na análise postural, com domínio do protocolo e de manuseio do software de avaliação, que analisou e estabeleceu as relações, alterações e adaptações posturais.

Os dados foram inseridos em uma planilha para análise descritiva por meio da obtenção dos valores de média, desviopadrão e análise da distribuição de frequência. Os dados foram extraídos no próprio Excel.

Este projeto foi registrado no Comitê de Ética em Pesquisa sob o CAAE ${ }^{\circ}$ 03250818.4.0000.8093 e aprovado pelo parecer $\mathrm{n}^{\mathrm{o}}$ 3.058.749. Todos os participantes assinaram o Termo de Consentimento Livre e Esclarecido, atendendo à Resolução ${ }^{\circ}$ 466/2012 do Conselho Nacional de Saúde e suas complementares.

\section{RESULTADOS}

Todos os participantes relataram possuir treinamento formal para o canto, desenvolvido com professor de canto $(100 \%)$, associado a preparador vocal $(50,0 \%)$ ou a músico $(33,3 \%)$ ou a coaching vocal (16,7\%). Dentre os participantes, $22,2 \%$ relataram trabalhar exclusivamente com o canto e $77,8 \%$ exerciam atividades de canto associadas a outras atividades com elevada demanda vocal, como professor, professor de canto, música de rua ou teatro.

Todas as mulheres, $66,7 \%$ da amostra, já haviam procurado profissionais por conta de alterações vocais na voz cantada
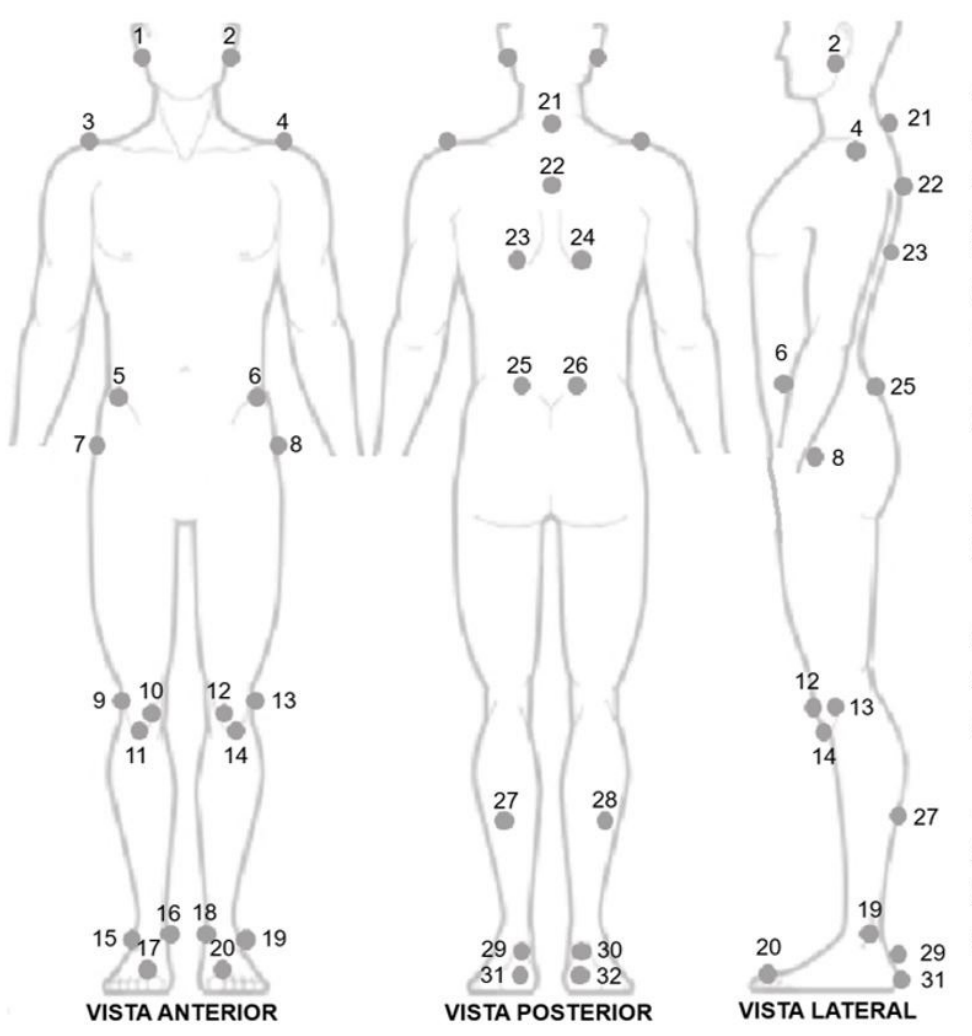

Vista Anterior

Alinhamento horizontal da cabeça: (1-2)

Alinhamento horizontal dos acrômios: (3-4)

Alinhamento horizontal das espinhas iliacas anterossuperiores: (5-6)

Ângulo entre os dois acrômios e as duas espinhas iliacas anterossuperiores: D (3-5) e E (4-6)

Ângulo frontal do membro inferior: D (7-9-15) e E (8-13-19)

Diferença no comprimento dos membros inferiores: D (5-16) e E (6-18)

Alinhamento horizontal das tuberosidades das tíbias: (11-14)

Ângulo Q: D (5-10 e 10-11) e E (6-12 e 12-14)

Vista Posterior

Assimetria horizontal da escápula em relação à T3: D (24-22) e E (23-22)

Ângulo perna/retropé: D (27-29-31) e E (28-30-32)

Vista Lateral Esquerda

Alinhamento horizontal da cabeça (C7): (2-21)

Alinhamento vertical da cabeça (acrômio): (4-2)

Alinhamento vertical do tronco: (4-8)

Ângulo do quadril (tronco e coxa): (4-8-19)

Alinhamento vertical do corpo: (4-19)

Alinhamento horizontal da pélvis: (6-25)

Ângulo do joelho: (8-13-19)

Ângulo do tornozelo: (13-19)

Figura 1. Marcação dos pontos anatômicos utilizados para a avaliação postural pelo software SAPO nas vistas anterior, posterior e lateral esquerda. 
$(50,0 \%)$ ou nas vozes cantada e falada simultaneamente $(50,0 \%)$. Os profissionais de suporte mais citados foram o professor de canto e o fonoaudiólogo $(66,7 \%$, cada), seguido do otorrinolaringologista $(50,0 \%)$, e a queixa mais frequente foi a rouquidão $(66,7 \%)$.

Todos os participantes relataram abusos ou maus usos da voz, como uso excessivo $(50,0 \%)$, cantar fora da tessitura $(33,3 \%)$, esforço vocal $(16,7 \%)$ e falta de aquecimento vocal $(16,7 \%)$. Entretanto, $88,9 \%$ relataram realizar aquecimento e desaquecimento vocais com exercícios de trato vocal semiocluído (sons nasais, fricativos sonoros, sopro e som agudo, bocca chiusa, vibração de língua e de lábios), vocal fry, vocalizes ascendentes e descendentes e bocejos.

A Tabela 1 apresenta os valores individuais dos parâmetros acústicos da voz dos participantes deste estudo.

A Tabela 2 apresenta os escores da APA da voz dos participantes deste estudo.

Os dados de avaliação postural sugerem que houve desvio de postura em todos os participantes, uma vez que nenhum deles se enquadrou entre os valores referência propostos pela análise realizada (Tabela 3).

Tabela 1. Dados da análise acústica da voz dos participantes do estudo

\begin{tabular}{|c|c|c|c|c|c|c|}
\hline IND & TMF (s) & $\mathrm{FO}(\mathrm{Hz})$ & $10(\mathrm{~dB})$ & Jitter (\%) & Shimmer (dB) & PHR \\
\hline 1 & 13,3 & 247,7 & 64,2 & 0,238 & 1,517 & 21,7 \\
\hline 2 & 14,0 & 223,7 & 66,6 & 0,110 & 0,903 & 31,5 \\
\hline 3 & 7,1 & 198,4 & 65,8 & 0,306 & 2,401 & 19,2 \\
\hline 4 & 8,5 & 239,5 & 67,6 & 0,241 & 1,944 & 20,6 \\
\hline 5 & 10,5 & 239,2 & 68,6 & 0,149 & 0,998 & 25,4 \\
\hline 6 & 7,1 & 219,8 & 66,9 & 0,123 & 0,996 & 28,3 \\
\hline 7 & 22,0 & 115,6 & 59,8 & 0,230 & 2,673 & 18,6 \\
\hline 8 & 17,0 & 95,0 & 68,0 & 0,196 & 2,168 & 18,1 \\
\hline 9 & 18,4 & 104,7 & 67,0 & 0,141 & 1,561 & 21,9 \\
\hline
\end{tabular}

Legenda: IND = indivíduo; TMF = tempo máximo fonatório; F0 = frequência fundamental; 10 = intensidade

vocal; $\mathrm{PHR}=$ proporção harmônico-ruído. Mulheres, participantes 1 a 6 . Homens, participantes 7 a 9

Tabela 2. Descrição pelos juízes dos settings não neutros da qualidade vocal dos participantes do estudo, utilizando o protocolo VPAS-PB

\begin{tabular}{|c|c|c|c|c|c|c|c|c|c|}
\hline Settings/Ajustes & 1 & 2 & 3 & 4 & 5 & 6 & 7 & 8 & 9 \\
\hline \multicolumn{10}{|c|}{ A. ELEMENTOS DO TRATO VOCAL } \\
\hline \multicolumn{10}{|l|}{ 1. Labial } \\
\hline Arredondamento/Protrusão & 3 & - & - & 3 & - & - & - & - & - \\
\hline Estiramento & - & - & 3 & - & 3 & 3 & 1 & - & - \\
\hline Extensão diminuída & 2 & - & - & - & - & - & - & - & - \\
\hline Extensão aumentada & - & - & - & 3 & - & - & - & 3 & - \\
\hline \multicolumn{10}{|l|}{ 2. Mandibular } \\
\hline Mandíbula fechada & - & - & 3 & - & - & 2 & - & - & - \\
\hline Extensão diminuída & 2 & - & 3 & - & - & - & - & - & - \\
\hline Extensão aumentada & - & - & - & - & - & - & - & 2 & - \\
\hline \multicolumn{10}{|l|}{ 3. Língua ponta/lâmina } \\
\hline Avançada & - & 1 & - & - & - & 3 & - & - & - \\
\hline Recuada & - & - & 3 & - & - & - & 1 & - & 2 \\
\hline \multicolumn{10}{|l|}{ 4. Corpo de língua } \\
\hline Recuado & 2 & - & - & - & - & - & - & - & - \\
\hline Elevado & 2 & - & 3 & 3 & 2 & - & - & - & 3 \\
\hline Extensão diminuída & - & - & - & - & 2 & - & - & - & - \\
\hline \multicolumn{10}{|l|}{ 5. Faringe } \\
\hline Constrição & - & - & 3 & 1 & 3 & 3 & - & 2 & 1 \\
\hline Expansão & - & - & - & - & - & - & - & 1 & - \\
\hline \multicolumn{10}{|l|}{ 6. Velofaringe } \\
\hline Nasal & - & 2 & 2 & 1 & 3 & 3 & - & 3 & 2 \\
\hline \multicolumn{10}{|l|}{ 7. Altura da laringe } \\
\hline Elevada & - & - & - & 3 & 2 & 2 & - & - & - \\
\hline Abaixada & - & - & 3 & - & - & - & - & - & - \\
\hline \multicolumn{10}{|l|}{ B. TENSÃO MUSCULAR GERAL } \\
\hline \multicolumn{10}{|l|}{ 8. Tensão do trato vocal } \\
\hline Hiperfunção & - & - & 3 & 2 & 3 & 3 & - & - & - \\
\hline \multicolumn{10}{|l|}{ 9. Tensão Laríngea } \\
\hline Hiperfunção & 1 & - & 3 & 3 & 3 & 3 & - & 3 & 3 \\
\hline
\end{tabular}


Tabela 2. Continuação...

\begin{tabular}{|c|c|c|c|c|c|c|c|c|c|}
\hline Settings/Ajustes & 1 & 2 & 3 & 4 & 5 & 6 & 7 & 8 & 9 \\
\hline Hipofunção & - & 3 & - & - & - & - & - & - & - \\
\hline \multicolumn{10}{|l|}{ 10. Modo de fonação } \\
\hline Crepitância (creak) & - & - & - & - & - & - & $\mathrm{N}$ & - & - \\
\hline Voz crepitante (creaky voice) & - & - & - & - & - & - & - & 3 & 3 \\
\hline \multicolumn{10}{|l|}{ 11. Fricção laríngea } \\
\hline Voz soprosa & 1 & 1 & 3 & 2 & 2 & 3 & 1 & 2 & 2 \\
\hline \multicolumn{10}{|l|}{ 12. Irregularidade laríngea } \\
\hline Voz áspera & - & - & 3 & 1 & - & 3 & 1 & - & 3 \\
\hline Ocorrências de curto prazo & - & - & - & - & - & - & - & - & - \\
\hline Quebras & - & - & 1 & - & - & - & - & - & - \\
\hline Instabilidade & - & - & I & $\mathrm{i}$ & $\mathrm{i}$ & $\mathrm{i}$ & $\mathrm{i}$ & - & $\mathrm{P}$ \\
\hline Elevado & - & - & - & 3 & 3 & 3 & - & - & - \\
\hline Extensão diminuída & 2 & - & - & - & - & - & - & 2 & - \\
\hline Variabilidade abaixada & - & 1 & - & - & - & - & - & - & - \\
\hline \multicolumn{10}{|l|}{ 14. Loudness } \\
\hline Diminuído & - & 1 & - & - & - & - & - & - & - \\
\hline Variabilidade abaixada & - & 3 & - & - & - & - & - & - & - \\
\hline \multicolumn{10}{|l|}{ E. ORGANIZAÇÃO TEMPORAL } \\
\hline \multicolumn{10}{|l|}{ 15. Tempo } \\
\hline Continuidade interrompida & - & 2 & - & - & - & - & 1 & - & - \\
\hline Tempo de elocução rápido & - & - & 3 & - & 3 & - & - & - & - \\
\hline Tempo de elocução lento & - & 3 & - & - & - & - & 1 & - & - \\
\hline
\end{tabular}

Legenda: $\mathrm{N}$ = não neutro; $\mathrm{P}$ = presente; $\mathrm{i}$ = intermitente. Mulheres, participantes 1 a 6 . Homens, participantes 7 a 9

Tabela 3. Descrição da avaliação postural individual realizada pelo software SAPO dos participantes do estudo. Todos os valores são expressos em graus

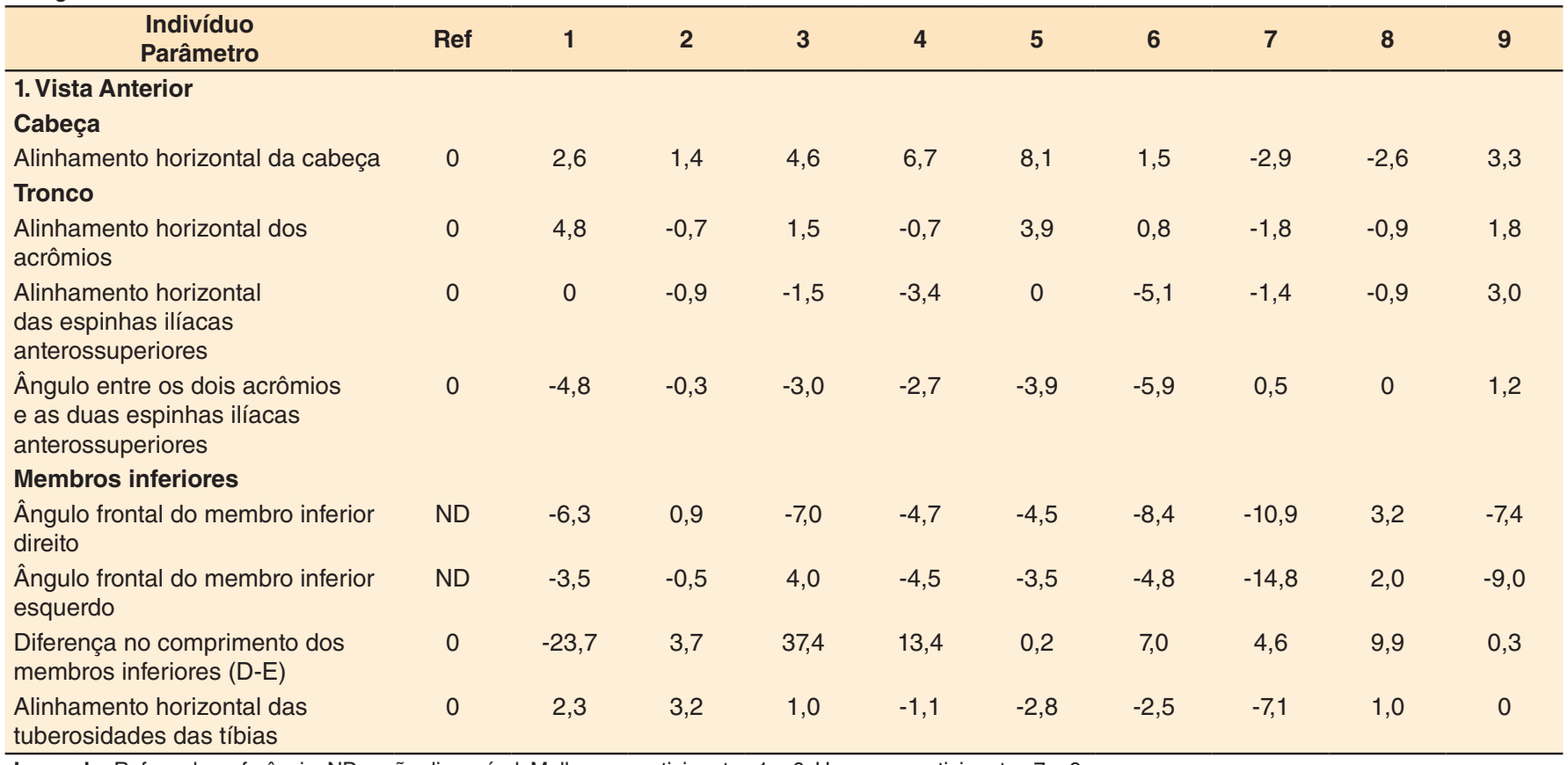

Legenda: Ref = valor referência; ND = não disponível. Mulheres, participantes 1 a 6 . Homens, participantes 7 a 9 
Tabela 3. Continuação...

\begin{tabular}{|c|c|c|c|c|c|c|c|c|c|c|}
\hline $\begin{array}{l}\text { Indivíduo } \\
\text { Parâmetro }\end{array}$ & Ref & 1 & 2 & 3 & 4 & 5 & 6 & 7 & 8 & 9 \\
\hline Ângulo $Q$ direito & 15 & 37,7 & 29,8 & 20,6 & 25,9 & 35,7 & 46,6 & 2,9 & 23,2 & 16,1 \\
\hline Ângulo $Q$ esquerdo & 15 & 21,7 & 17,2 & $-10,3$ & 17,6 & 47,5 & 33,5 & 10,5 & 12,9 & 1,2 \\
\hline \multicolumn{11}{|l|}{ 2. Vista Posterior } \\
\hline \multicolumn{11}{|l|}{ Tronco } \\
\hline $\begin{array}{l}\text { Assimetria horizontal da escápula } \\
\text { em relação à T3 }\end{array}$ & 0 & 30,8 & 42,6 & 29,9 & 18,2 & 56,3 & 5,1 & 7,3 & 35,3 & 27,6 \\
\hline \multicolumn{11}{|l|}{ Membros inferiores } \\
\hline Ângulo perna/retropé direito & ND & 1,6 & 3,6 & 9,0 & 8,0 & 7,4 & 2,7 & 18,9 & 6,7 & 13,8 \\
\hline Ângulo perna/retropé esquerdo & ND & 5,8 & 1,1 & $-10,7$ & 5,1 & 8,5 & 28,5 & 3,4 & 9,8 & $-2,9$ \\
\hline \multicolumn{11}{|l|}{ 3. Vista Lateral Esquerda } \\
\hline \multicolumn{11}{|l|}{ Cabeça } \\
\hline $\begin{array}{l}\text { Alinhamento horizontal da cabeça } \\
\text { (C7) }\end{array}$ & ND & 40,3 & 38,0 & 49,4 & 46,4 & 45,0 & 50,7 & 37,9 & 56,6 & 38,9 \\
\hline $\begin{array}{l}\text { Alinhamento vertical da cabeça } \\
\text { (acrômio) }\end{array}$ & 0 & 29,2 & 9,6 & 13,8 & 15 & 19,7 & 23,1 & 24,1 & 7,4 & 20,8 \\
\hline \multicolumn{11}{|l|}{ Tronco } \\
\hline Alinhamento vertical do tronco & ND & $-7,4$ & 0,9 & $-2,1$ & $-7,1$ & -6 & $-5,9$ & $-3,6$ & $-4,8$ & 1,2 \\
\hline Ângulo do quadril (tronco e coxa) & ND & $-23,6$ & 2,5 & 2,3 & $-10,8$ & $-14,9$ & $-7,4$ & $-9,1$ & $-8,0$ & $-5,4$ \\
\hline Alinhamento vertical do corpo & ND & 2,9 & 2,9 & 2,3 & 0,5 & 2,3 & 0,7 & 3,5 & 1,6 & 4,5 \\
\hline Alinhamento horizontal da pélvis & ND & $-13,0$ & $-35,6$ & $-16,1$ & $-22,0$ & $-14,0$ & $-19,1$ & $-16,4$ & $-13,8$ & $-10,6$ \\
\hline \multicolumn{11}{|l|}{ Membros inferiores } \\
\hline Ângulo do joelho & ND & $-12,3$ & 12 & 21,7 & 4,3 & $-1,5$ & 8,6 & 5,5 & 8,0 & 0,8 \\
\hline Ângulo do tornozelo & ND & 86,1 & 79,6 & 72,7 & 82,0 & 82,6 & 79,9 & 79,0 & 78,8 & 82,6 \\
\hline \multicolumn{11}{|l|}{ 4. Centro de gravidade } \\
\hline Assimetria no plano frontal (\%) & - & 25,8 & 0,7 & 10,2 & 8,4 & 0,9 & 4,9 & $-17,3$ & $-4,5$ & 9,1 \\
\hline Assimetria no plano sagital (\%) & - & 62,8 & 41,5 & 49,2 & 37,3 & 56,5 & 39,6 & 59,6 & 43,0 & 50,5 \\
\hline $\begin{array}{l}\text { Posição da projeção do CG } \\
\text { relativo à posição média dos } \\
\text { maléolos (plano frontal) }(\mathrm{cm})\end{array}$ & - & 25,8 & $-0,6$ & 12,8 & $-8,7$ & 0,8 & 4,6 & $-23,7$ & $-5,9$ & 12,6 \\
\hline $\begin{array}{l}\text { Posição da projeção do CG } \\
\text { relativo à posição média dos } \\
\text { maléolos (plano lateral) }(\mathrm{cm})\end{array}$ & - & 75,3 & 52,7 & 57,5 & 44,7 & 60,5 & 44,8 & 85,2 & 57,2 & 72,7 \\
\hline
\end{tabular}

\section{DISCUSSÃO}

Neste estudo conseguiu-se a participação efetiva de um pequeno grupo de cantores, seis mulheres e três homens, cujo potencial vocal é reconhecido e valorizado socialmente e cujas carreiras estão consolidadas localmente.

Constatou-se, por meio da avaliação perceptivoauditiva da voz que todos os participantes apresentaram algum ajuste não-neutro de trato vocal. Todos os participantes apresentaram qualidade vocal soprosa, cinco, voz áspera, e dois, voz crepitante, com presença de suporte respiratório inadequado. Esses achados podem sugerir que as alterações vocais observadas no grupo decorrem de ajustes vocais inadequados e não, necessariamente, por alterações laríngeas, o que pode justificar o fato de cantores experientes não referirem ou não perceberem as alterações de qualidade vocal.

O suporte respiratório inadequado pode ocasionar um antagonismo entre a pressão subglótica e a resistência glótica. A alteração da vibração das pregas vocais pode ser produzida pela presença de lesões estruturais, pela existência de hipertonia da musculatura laríngea ou pela deficiência da coaptação glótica ${ }^{(10)}$. A soprosidade e a tensão são qualidades vocais nas quais se observa grande atividade dos músculos supra-hioideos, que podem elevar a laringe e fazer com que esse som seja produzido em uma frequência mais aguda, com uma projeção reduzida e esforço na região laringofaríngea ${ }^{(11)}$ e podem estar associados com alterações na postura da cabeça e pescoço, como observado no grupo estudado, cujos dados de APA sugerem que há uma hiperfunção do trato vocal, com presença de tensão laríngea de grau moderado ${ }^{(3)}$. Além disso, podem ser observados desvios de loudness, pitch, sinais e sintomas laríngeos, como fadiga vocal e afonia, o que pode ser uma forma de o corpo demonstrar que existe alteração em determinado segmento, gerando compensações e sobrecargas em sua funcionalidade ${ }^{(12)}$.

À medida em que o pitch muda de grave para agudo, a coluna cervical altera sua curvatura de lordose para cifose, encurtando a via aérea e elevando laringe, epiglote e palato mole em relação à coluna cervical; a distância entre o esterno e a laringe se torna maior e a distância entre o hioide e a laringe diminui. Assim, a postura pode ter um efeito direto sobre o esforço vocal, ainda que não haja carga vocal. Mesmo em mudanças sutis no posicionamento de cabeça e no equilíbrio pode existir um impacto significativo no movimento laríngeo e em sua eficiência. A postura de boca aberta, por exemplo, geralmente está relacionada a mudanças efetivas no comportamento vocal, englobando elevação de 
F0, voz mais clara e melhor estabilidade na fonação ${ }^{(13)}$. Neste estudo, a maioria dos participantes apresentou hipercifose com anteriorização de cabeça, associada à escoliose em cinco deles e à retração peitoral em três, o que a literatura refere como comum entre cantores e locutores, associando com protrusão de ombros e extensão da coluna cervical ${ }^{(5)}$.

Os músculos intrínsecos da laringe são responsáveis pela movimentação das cartilagens aritenoideas, e, portanto, pela adução, abdução e tensão das pregas vocais. Já a musculatura extrínseca mantém a laringe em posição natural e estável, possibilitando que a musculatura intrínseca se contraia de forma livre e sem perturbações ${ }^{(3,13)}$. No alinhamento adequado da coluna torácica e cervical, os músculos esternocleidomastoideo e escaleno têm sua atividade diminuída, estabilizando a cervical, e, em consequência, permitindo a base para a mobilidade cervical e laríngea ${ }^{(14)}$.

A forma como o indivíduo respira também influencia tanto na postura quanto na voz. A incoordenação respiratória ou o padrão oral de respiração no repouso podem levar a alterações da postura corporal, as quais, por sua vez, afetam o posicionamento da laringe. Na respiração costal, por exemplo, há maior atividade do músculo esternocleidomastoideo. $\mathrm{E}$, quanto mais presente a hipertonicidade da musculatura e a postura corporal inadequada, ou mesmo a combinação de ambas, maiores serão as queixas sobre as desvantagens vocais. Uma tensão cervical ou escapular do tipo assimétrica pode levar a um aumento da lordose cervical e impactar no processo fonatório ${ }^{(15)}$.

$\mathrm{O}$ ato de cantar exige coordenação entre o sistema respiratório e fonatório. Regularmente a expiração favorece a capacidade do cantor para sustentar uma nota. Durante uma sustentação da nota musical fica perceptível muitas das características da voz e suas limitações na realização/utilização das técnicas. A não sustentação precisa da nota musical, no fim de uma estrofe, retrata uma sensação de fadiga e um reduzido controle vocal, o que pode comprometer a intenção comunicativa da música e o surgimento das emoções que esta causaria no ouvinte. Desse modo, o TMF é uma forma de identificar como a laringe se comporta frente à força aerodinâmica produzida durante a passagem do ar advindo dos pulmões, sendo o tempo de emissão diretamente proporcional à capacidade do indivíduo em controlar a saída do fluxo de ar expirado ${ }^{(16)}$.

A avaliação visual que fornece uma "leitura corporal" básica realizada por diferentes profissionais, pode ser enriquecida pela internalização do conceito de "tensegridade", uma vez que os terapeutas buscam o alinhamento biomecânico e outras formas de eficiência do movimento, assim como a "alfabetização cinestésica" e o alívio psicossomático. Partindo deste conceito, bem como de suas propriedades distributivas, acomodando tensão ou trauma local pela dispersão mediante pequenos ajustes ao longo de todo o sistema, à medida em que se resolvem padrões disfuncionais, aproxima-se mais de um equilíbrio, trazendo a eficiência e o alívio desejados ${ }^{(7)}$.

O equilíbrio entre as estruturas de suporte deveria envolver uma quantidade mínima de esforço e sobrecarga, com uma máxima eficiência do corpo, buscando sua coordenação para a postura ideal do indivíduo, seja no movimento ou no repouso. Para o equilíbrio, pois, é importante a manutenção do alinhamento biomecânico, ou seja, o equilíbrio entre as cinturas escapular e pélvica, para que se obtenha um melhor posicionamento corporal global pelas relações entre o tônus corporal e a força da gravidade; posição da cabeça em harmonia com a articulação temporomandibular e a cintura pélvica; distribuição adequada do peso nos dois lados do corpo; projeção de todo o peso do corpo no solo, refletindo-se sobre o centro dos pés, visão, propriocepção e sistema vestibular ${ }^{(17-19)}$.

Para obter o equilíbrio, deve-se considerar tanto as estruturas dinâmicas como estáticas responsáveis pelo equilíbrio postural. Os músculos propiciam forças dinâmicas de oposição a movimentos de extensão e flexão causados pelo torque gravitacional das articulações e necessitam de um sistema nervoso intacto para proporcionar ofeedback sensório-motor. As estruturas ósseas e ligamentos proporcionam uma tensão passiva nas articulações e apoio para sustentação de peso na postura ereta. Em equilíbrio, a linha de gravidade passa nas proximidades ou através dos eixos de rotação das articulações e as forças compressivas são otimamente distribuídas sobre as superfícies de sustentação de peso ${ }^{(17-19)}$.

A adequação do alinhamento postural favorece, então, a melhora no alinhamento da cabeça em pessoas que apresentam condições clínicas como tensão exacerbada dos músculos trapézios (fibras superiores), temporais e masseteres, disfunção temporomandibular e desvios de postura. Isso demonstra que os desvios posturais geram tensão sobre as cadeias musculares e, por conseguinte, sobre os músculos mastigatórios e do pescoço, podendo interferir nas funções da laringe ${ }^{(12)}$

A posição inadequada da cabeça muda as relações biomecânicas craniofaciais e craniomandibulares e sofre influência do crescimento e da postura corporal do indivíduo, sendo de extrema importância que se observe os desvios funcionais desde seu início ${ }^{(7,20)}$.

Conjugar o estudo postural com a análise vocal foi um grande desafio para o grupo, haja vista o conhecimento dos padrões posturais, mas o desconhecimento de como analisálos sem a influência de uma "leitura corporal" meramente visual. A interação com o profissional de Educação Física e a identificação da possibilidade de uso da biofotogrametria, cuja confiabilidade foi reconhecida, agregou a possibilidade de uma avaliação objetiva dos desvios e assimetrias posturais, uma vez que seu registro evidencia as interrelações entre as partes do corpo e as mínimas alterações posturais, cuja mensuração é de difícil avaliação ${ }^{(21)}$.

Deve-se considerar que a percepção que muitas pessoas têm de seus desvios posturais é quase sempre limitada, pois as alterações na postura de cabeça, pescoço, ombros, abdômen e quadril por vezes estão integradas ao sistema de movimento adotado pelo indivíduo que passam a ser habituais e tidas como parte de sua constituição física ${ }^{(22)}$. Desta forma, pode-se avaliar e sugerir a relação existente entre a postura e os músculos extrínsecos da laringe, estabelecendo que na postura ereta a produção do som vocal é melhorada ${ }^{(5,13)}$.

A avaliação e/ou a visualização gráfica da voz permite a obtenção de parâmetros sonoros específicos, importantes no auxílio e na compreensão do mecanismo da fonação, além de permitir a inferência dos mecanismos presentes nos diferentes distúrbios da voz ${ }^{(23)}$ e, como já discutido que uma boa postura tem papel fundamental na adequada qualidade da voz ${ }^{(3)}$, na mesma medida em que uma postura incorreta pode provocar dor e alteração na função de diferentes sistemas, poderia se relacionar a tensegridade do corpo, quando descreve um princípio de relação estrutural em que a forma da estrutura é garantida pelos comportamentos tensionais, ou seja, se qualquer dos elementos de tensegridade se romper, esse equilíbrio dinâmico é perturbado e a estrutura vai mudar de forma e atingir ou buscar outro ponto de equilíbrio ${ }^{(7)}$. 
Um exemplo disso é a protrusão da cabeça, que pode gerar uma disfuncionalidade dos músculos anteriores do pescoço, como o esternocleidomastóideo, os supra- e infra-hioideos e o escaleno, o que, por conseguinte, provoca alteração na funcionalidade da musculatura extrínseca de laringe e faz surgir inadaptações e variações na produção vocal ${ }^{(21)}$. E, pode-se considerar que estes padrões posturais podem estar associados às lordose ou cifose excessivas, gerando uma compensação ou adaptação muscular na laringe e no pescoço, que pode se refletir como uma das causas de problemas com o controle do pitch em cantores ${ }^{(15)}$.

O encurtamento dos músculos da região peitoral e o encurtamento adaptativo da musculatura do abdômen, quando o indivíduo assume uma postura cifótica torácica, traz como consequência o impedimento dos músculos trapézio médio e romboides reposicionarem os ombros de maneira correta, comprometendo seu alinhamento ${ }^{(4)}$.

A tensão exacerbada da musculatura cervical interfere no equilíbrio corporal, pois afeta a posição do aparelho vestibular e dos músculos responsáveis pela regulação de movimento ocular, gerando menor amplitude dos movimentos de pescoço e anteriorização de cabeça, deixando a musculatura infrahioideia mais tensa e tracionando a mandíbula posteriormente ${ }^{(12)}$. Durante uma extensão cervical a musculatura anterior do pescoço se estende enquanto os músculos trapézios e elevadores da mandíbula (musculatura suboccipital) se encurtam. Assim, ao se assumir essa postura os músculos anteriores enfraquecem, enquanto o grupo suboccipital se encurta, o que pode provocar mudança de forma da laringe, principalmente o seu estreitamento, intensificando a adução das pregas vocais e modificando a ressonância da voz ${ }^{(24)}$.

Assim, é essencial que se crie uma consciência postural, por meio do autoconhecimento do corpo e da postura, de acordo com as possibilidades biomecânicas individuais. Exercícios como relaxamento geral, regulação da respiração, movimentação articular, percepção quanto ao equilíbrio alinhado, percepções de contato e de sensações são recomendados ${ }^{(2)}$.

Embora seja uma pesquisa exploratória inicial sobre as possibilidades de coocorrência de alterações ou adaptações posturais com a qualidade vocal avaliada, pôde-se observar que em todos os casos analisados houve presença de alterações posturais e de parâmetros vocais, o que evidenciou que a voz é produto do corpo inteiro e que, mesmo profissionais do canto, atentos à qualidade de suas vozes, não percebem ou não valorizam os impactos e desvantagens decorrentes, muito provavelmente, da adoção de posturas ou posições adaptadas ou compensatórias.

A conscientização destes profissionais da voz, principalmente daqueles que se movem, dançam e necessitam de fortes intensidades durante suas performances, trará inúmeros benefícios e um impacto significativo, proporcionando uma voz clara, livre e que atinja o objetivo final que é de emocionar e se fazer perceber pelo público.

\section{CONCLUSÃO}

Foram identificadas e descritas as alterações de postura e de qualidade de voz em um grupo de cantores populares. Todos os participantes apresentaram adaptações e/ou alterações posturais, ajustes não-neutros à fonação e variação nos parâmetros acústicos e perceptivoauditivos, sugerindo que as alterações vocais e posturais coocorrem nestes indivíduos.

\section{REFERÊNCIAS}

1. Mello EL, Ballestero LRB, Silva MAA. Postura corporal, voz e autoimagem em cantores líricos. Per Musi. 2015;31(31):74-85. http:// dx.doi.org/10.1590/permusi2015a3104.

2. Salve MGC, Bankoff ADP. Body posture - a problem that afflicts workers. Rev Bras Saúde Ocup. 2003;3(105-106):91-103. http:// dx.doi.org/10.1590/S0303-76572003000100010.

3. Nacci A, Fattori B, Mancini V, Panicucci E, Matteucci J, Ursino F, et al. Posturographic analysis in patients with dysfunctional dysphonia before and after speech therapy/rehabilitation treatment. Acta Otorhinolaryngol Ital. 2012 Abr;32(2):115-21. PMid:22767973.

4. Wilson Arboleda BM, Frederick AL. Considerations for maintenance of postural alignment for voice production. J Voice. 2008 Jan;22(1):90-9. http://dx.doi.org/10.1016/j.jvoice.2006.08.001. PMid:16978836.

5. Carneiro PR, Teles LCS. Influence of postural alterations, followed by computadorized photogrammetry, in the voice production. Fisioter Mov. 2012 Mar;25(1):13-20. http://dx.doi.org/10.1590/S010351502012000100002 .

6. Mello EL, Silva MAA, Ferreira LP, Herr M. Lyric singer voice and motor coordination: an intervention based on Piret and Béziers. Rev Soc Bras Fonoaudiol. 2009;14(3):352-61. http://dx.doi.org/10.1590/ S1516-80342009000300011.

7. Myers TW. Trilhos anatômicos: meridianos miofasciais para terapeutas manuais e do movimento. 3. ed. Barueri: Manole; 2016. 380 p.

8. Mello EL, Silva MAA. The singer's body: stretch, relax or warmup? Rev CEFAC. 2008 Dez;10(4):548-56. http://dx.doi.org/10.1590/ S1516-18462008000400015.

9. Camargo Z, Madureira S. Voice quality analysis from a phonetic perspective: Voice Profile Analysis Scheme (VPAS) profile for Brazilian Portuguese. In: Proceedings of the 4th International Conference on Speech Prosody; 2008 Mar 6-9; Campinas. São Paulo: Speech Prosody; 2008.

10. Cielo CA, Conterno G, Carvalho CDM, Finger LS. Disfonias: relação S/Z e tipos de voz. Rev CEFAC. 2008 Dez;10(4):536-47. http://dx.doi. org/10.1590/S1516-18462008000400014.

11. Costa DB, Lopes LW, Silva EG, Cunha GMS, Almeida LNA, Almeida AAF. The risk factors and emotional on the voice of teachers with and without vocal complaints. Rev CEFAC. 2013 Ago;15(4):1001-10.

12. Cielo CA, Christmann MK, Ribeiro VV, Hoffmann CF, Padilha JF, Steidl EMS, et al. Musculoskeletal stress syndrome, extrinsic laryngeal muscles and body posture: theoretical considerations. Rev CEFAC. 2014 Out;16(5):1639-49. http://dx.doi.org/10.1590/1982-0216201410613.

13. Cardoso R, Lumini-Oliveira J, Meneses RF. Associations between posture, voice, and dysphonia: a systematic review. J Voice. 2019 Jan;33(1):124.e1-12. http://dx.doi.org/10.1016/j.jvoice.2017.08.030. PMid:29032127.

14. Staes FF, Jansen L, Vilette A, Coveliers Y, Daniels K, Decoster W. Physical therapy as a means to optimize posture and voice parameters in student classical singers: a case report. J Voice. 2011 Maio;25(3):e91-101. http://dx.doi.org/10.1016/j.jvoice.2009.10.012. PMid:20207108.

15. Kooijman PGC, de Jong FICRS, Oudes MJ, Huinck W, van Acht $\mathrm{H}$, Graamans K. Muscular tension and body posture in relation to voice handicap and voice quality in teachers with persistent voice 
complaints. Folia Phoniatr Logop. 2005;57(3):134-47. http://dx.doi. org/10.1159/000084134. PMid:15914997.

16. Silva EGF, Luna CLC. Perceptual vocal pattern analysis of singers from kitschy musical style in Recife. Rev CEFAC. 2009 Set;11(3):457-64.

17. Wang J-S, Cho K-H, Park S-J. The immediate effect of diaphragm taping with breathing exercise on muscle tone and stiffness of respiratory muscles and SpO2 in stroke patient. J Phys Ther Sci. 2017 Jun;29(6):970-3. http://dx.doi.org/10.1589/jpts.29.970. PMid:28626302.

18. Sze WP, Yoon WL, Escoffier N, Rickard Liow SJ. Evaluating the training effects of two swallowing rehabilitation therapies using surface electromyography: Chin Tuck Against Resistance (CTAR) exercise and the shaker exercise. Dysphagia. 2016 Abr 2;31(2):195-205. http:// dx.doi.org/10.1007/s00455-015-9678-2. PMid:26837612.

19. Silva AP, Carvalho ARR, Sassi FC, Silva MAA. The taping method effects on the trapezius muscle in healthy adults. CoDAS. 2019;31(5):e20180077.
20. Silveira MC, Sígolo C, Quintal M, Sakano E, Tessitore A. Oral motricity photographic registration proposal. Rev CEFAC. 2006 Dez;8(4):485-92.

21. Bigaton DR, Silvério KCA, Berni KCS, Distefano G, Forti F, Guirro RRJ. Craniocervical posture in dysphonic women. Rev Soc Bras Fonoaudiol. 2010;15(3):329-34. http://dx.doi.org/10.1590/S151680342010000300004 .

22. Machado PG, Mezzomo CL, Badaró AFV. Body posture and the stomatognathic functions in mouth breathing children: a literature review. Rev CEFAC. 2012 Abr 26;14(3):553-65. http://dx.doi. org/10.1590/S1516-18462012005000033.

23. Medina V, Simões-Zenari M, Nemr NK. Acoustic voice analysis: effect of an audio-visual training for speech therapy undergraduates. Audiol Commun Res. 2015 Jun;20(2):123-9. http://dx.doi.org/10.1590/ S2317-64312015000200001419.

24. Behlau P, Pontes P. Avaliação e tratamento das disfonias. São Paulo: Lovise; 1995. 